\title{
Role of surface structure in photoelectron emission from solid Ne: impurities, defect and low-coordinated sites
}

\author{
Yu.A. Dmitriev \\ A.F. Ioffe Physico-Technical Institute, 26 Politekhnicheskaya Str., St. Petersburg 194021, Russia \\ E-mail: dmitrievyurij@gmail.com \\ Received March 28, 2012, revised May 4, 2012
}

\begin{abstract}
Electron emission was obtained from a solid Ne sample growing from the gas phase on a low temperature substrate. The surface of the sample was irradiated by the light of an open-source microwave discharge running in the gaseous Ne. The irradiation produces electron emission from the sample. A second gas flow of $\mathrm{D}_{2}$ was, simultaneously, passed onto the substrate avoiding the discharge zone. Free electrons ejected into a vacuum chamber during the sample growth were detected by means of the electron cyclotron resonance technique. The emission shows nonmonotonic dependence on the impurity $\mathrm{D}_{2}$ concentration. At small concentration, the electron yield is found to increase significantly at increasing the dopant flow rate, while, at moderate concentrations, the yield tends to decrease with increasing the flow rate. A tentative explanation of the observed effect is presented based on the exceptional properties which the neon-hydrogen solid mixture is believed to have.
\end{abstract}

PACS: $52.50 . \mathrm{Sw}$ Plasma heating by microwaves; ECR, LH, collisional heating;

79.60.-i Photoemission and photoelectron spectra;

79.75. $+\mathrm{g}$ Exoelectron emission.

Keywords: Ne solid, ECR, photoelectron emission, surface impurities and traps.

\section{Introduction}

In earlier studies [1,2], an effect has been found that a small gas flow of He provided onto the cold substrate where the gaseous $\mathrm{Ne}$ supplied through a gas discharge tube was condensed suppressed the electron photoemission from the solid Ne. The electron emission occurred from a solidified Ne layer subjected to irradiation from an electrodeless open discharge running in the gaseous Ne. The electron photoemission yield was measured using cyclotron resonance of free electrons (ECR) emitted from the solid Ne. In observing the resonance, we utilized a conventional X-band EPR spectrometer with the cylindrical microwave cavity. The arrangement of DC magnetic field and RF electric field was shown $[3,4]$ to be favorable for ECR line detection with the use of this cavity. The experimental results $[1,2]$ revealed an important role a surface plays in the process of photoelectron emission from rare gas solids. Also we were able to show that the bulk He impurities have negligible effect on the electron emission compared to the surface ones. To gain a better insight into the roles of bulk and surface impurities in the photoemission of free electrons, we tested this process using an impurity with lower ionization potential and readily adsorbed by a sample at liquid He temperatures. The molecular $\mathrm{CH}_{4}$ was uti- lized as such a probe [5]. The net effect of doping by the $\mathrm{CH}_{4}$ impurity on the photoelectron yield from the solid $\mathrm{Ne}$ was that the free electron emission decreased with increasing methane flow rate onto the Ne surface. Though the bulk $\mathrm{CH}_{4}$ impurities were believed to take some part in quenching emission, their influence was not elucidated. The major role was played by the surface $\mathrm{CH}_{4}$ molecules.

The similarity in solid $\mathrm{Ne}$ emission behavior under doping with such a different species like $\mathrm{He}$ and $\mathrm{CH}_{4}$ deserves special attention. Generally, it is thought that of guest atoms or molecules only those with positive electron affinity [6] could be a trap of the electron traveling through the lattice. The atomic He, however, is a particle with very small positive affinity, 0.0054 Ry [7] which is equal $0.073 \mathrm{eV}$, and the $\mathrm{CH}_{4}$ molecule has large negative electron affinity, $E a=-5 \mathrm{eV}[8,9]$. Thus, despite the affinities of different magnitudes and signs, both $\mathrm{He}$ and $\mathrm{CH}_{4}$ impurities turn out to be effective traps in solid $\mathrm{Ne}$, not allowing the electrons to escape into the vacuum. One of the explanations for the observed effect was that it could be related to the processes of electron trapping in insulator materials $[9,10]$. The authors established a relationship between the electron trap and the molecular properties of the material. They studied both physical (e.g., conformational disorder) and chemical defects (e.g., broken bonds and impurities) and 
showed that while typical physical trap energies were of the order of $0.15 \mathrm{eV}$ and all are less than $0.3 \mathrm{eV}$ (for polymeric insulators), the chemical defect trap energies reached about $1 \mathrm{eV}$ even for impurities with negative electron affinity for free molecules [10]. The trap energy, $E_{\text {trap}}$, is defined as the energy difference between the electron affinity of the system with and without the defect, so that

$$
E_{\text {trap }}=E A_{\text {defect }}-E A_{\text {reference }} \text {. }
$$

Therefore, even in case of negative electron affinity of the free impurity atom, the affinity for the adsorbed atom may be positive. Another explanation for the quenching mechanism of the electron emission by impurities was that the electron affinity of the impurity does not account for the effect, i.e., a surface impurity of any kind may prevent bulk electrons from appearing at the surface. To check this assumption, we carried out experiments with molecular $\mathrm{D}_{2}$ and provide, in this paper, detailed results of the study. The results turned out to be unexpected. It is generally agreed that the electron affinity of the $\mathrm{H}_{2}$ molecule is negative, though the exact magnitude is under question. Using the valence bond method Dalgarno and McDowell computed $E a=-3.6 \mathrm{eV}$ [11], while FisherHjalmars reported $-0.28 \mathrm{eV}$ [12]. Value of $-2.4 \mathrm{eV}$ is available from the book by $\mathrm{H}$. Stewart and W. Massey [13], and results between -2 and $-1 \mathrm{eV}$ are presented by J.K. Park depending on the calculation procedure, see [14] and references therein. With respect to the electron affinity, the molecular hydrogen is between $\mathrm{He}$ and $\mathrm{CH}_{4}$ impurities. It is worth noting that hydrogen coverage was found to change the electron affinity of the diamond crystal surface from positive of $+0.38 \mathrm{eV}$ to negative of $-1.27 \mathrm{eV}$ [15]. The hydrogenation was achieved by treating a diamond sample in hydrogen plasma. Amazingly, despite the difference in the solids under study and coverages used: solid $\mathrm{Ne}$ and $\mathrm{D}_{2}$ layer, in the present study, and diamond single crystal and $\mathrm{H}$ adatoms in Ref. 15, the effects which the coverages had on the electron emission turned out to be similar.

\section{Experimental}

The electrons escaping into the vacuum from the sample were observed through the electron cyclotron absorption using a conventional EPR device [4]. The setup and experimental procedure have been presented elsewhere $[4,16]$. Briefly, they were as follows. The bottom of a quartz finger filled with liquid helium served as a low temperature substrate for the gases being condensed. The bottom was located at the center of the microwave cavity of an X-band EPR spectrometer. The cavity was evacuated and cooled externally with liquid nitrogen vapor providing a cavity temperature from 77 to $300 \mathrm{~K}$. An electrodeless high-frequency $(15 \mathrm{MHz})$ discharge operating in pulsed regime was excited in the gaseous $\mathrm{Ne}$ which was passed through a glass tube with an outlet of approximately 0.6 $\mathrm{mm}$ diameter. The products of the discharge entered the cavity and condensed on the finger bottom, forming $\mathrm{Ne}$ solid. The solid was subjected to the action of the irradiation from the outlet, which, thus, operated as an opendischarge source. The deuterium gas flow was supplied to the substrate by a quartz tube inserted into the cavity. The tube was placed outside the discharge zone. The end of the quartz tube was located close $(3 \mathrm{~mm})$ to the substrate. Thus two separate channels supplied gases under study to the substrate: the discharge channel and the matrix channel. Both gaseous flows were cooled with liquid nitrogen vapor prior to deposition. The substrate temperature was lowered down by pumping-out the liquid He bath. The base pressure in the experimental chamber was $2 \cdot 10^{-6}$ Torr. Pure gases were used with the following impurity contents: $0.004 \% \mathrm{Ne}$ and $0.3 \% \mathrm{D}_{2}$ (molecular hydrogen being the major impurity).

The working pressure of the microwave discharge was assessed as being of some $10^{2} \mathrm{~Pa}$ whereas the pressure in the cavity was in the tens of $\mathrm{mPa}$ range. The pressure difference was maintained by either small diameter of the outlet or differential pumping provided by both the cold surface of the quartz finger and the set-up pumping facilities. Since the spectra are taken when the discharge is running in the gaseous $\mathrm{Ne}$, the magnet is turned on and the strong external constant magnetic field of $\sim 0.33 \mathrm{~T}$ effects the discharge operation. The axis of the discharge tube is perpendicular to the magnetic field lines. In order to operate the discharge at low pressure and make it stable and prevent it's extinguish, we tried out different ways of coupling the high-frequency power into the discharge. Eventually we found that the best result was produced when the power was fed into two copper rods parallel to the tube axis and located in the plane perpendicular to the magnetic field lines. Thus the discharge operates as a windowless microwave source of the VUV radiation with differential pumping.

Table I. Energy positions of excitons in solid Ne from transmission data [18]. All energies are given in eV.

\begin{tabular}{c|c|c|c|c|c|c|c|c|c|c}
\hline \hline$n$ & \multicolumn{2}{|c|}{1} & \multicolumn{2}{c|}{2} & \multicolumn{2}{c|}{3} & \multicolumn{2}{c|}{4} & \multicolumn{2}{c}{5} \\
\hline$j$ & $3 / 2$ & $1 / 2$ & $3 / 2$ & $1 / 2$ & $3 / 2$ & $1 / 2$ & $3 / 2$ & $1 / 2$ & $3 / 2$ & $1 / 2$ \\
Energy position & 17.36 & 17.50 & 20.25 & 20.36 & 20.94 & 21.02 & 21.19 & - & 21.32 & - \\
\hline \hline
\end{tabular}

Here, $n$ is for the principle quantum number and $\mathrm{j}$ is for the momentum of the hole. 
Table 2. Wavelengths, emission intensities [19] and photon energies for the spectrum lines of neutral Ne atoms. The values reported in Ref. 19 represent the wavelength observed for a discharge in neon with the naturally occurring isotopic abundance. Here $\lambda$ is given in Á, while $E_{f}$ is in eV.

\begin{tabular}{c|c|c|c|c|c|c|c|c|c|c|c|c}
\hline$\lambda$ & 595.92 & 598.71 & 598.89 & 600.04 & 602.73 & 615.63 & 618.67 & 619.10 & 626.82 & 629.74 & 735.90 & 743.72 \\
$E_{f}$ & 20.81 & 20.71 & 20.71 & 20.66 & 20.57 & 20.14 & 20.04 & 20.03 & 19.78 & 19.69 & 16.85 & 16.67 \\
$\mathrm{I}$ & 30 & 20 & 10 & 20 & 40 & 50 & 50 & 40 & 60 & 60 & 300 & 120 \\
\hline
\end{tabular}

At the gas pressures in the range of tens or hundreds of Pascals, the main photon flux comes from the NeI radiation [17]. Once absorbed, the radiation may lead to an excitation creation in the solid Ne subjected to the VUV irradiation from the open discharge in gaseous Ne. Table 1 lists energy positions of exciton absorption bands in solid $\mathrm{Ne}$ [18]. Listed in Table 2 are wavelengths, intensities [19] and photon energies corresponding to the spectrum lines of neutral $\mathrm{Ne}$ atoms.

It is obvious from the Tables that the lines in the solid rare gases are shifted to higher energy as compared with the corresponding lines in the gas-phase. The blue shift decreases with increasing quantum number so that the series limit of the gas and that of the solid lie close together. Some gas-phase lines of the $2 s^{2} 2 p^{5} 4 d-2 s^{2} 2 p^{6}$, $2 s^{2} 2 p^{5} 5 s-2 s^{2} 2 p^{6}$ and $2 s^{2} 2 p^{5} 3 d-2 s^{2} 2 p^{6}$ transition arrays are within the absorption exciton bands of $\Gamma(4 s), \Gamma\left(4 s^{\prime}\right)$ and $\Gamma(5 s)$ transitions. Hence, the discharge irradiation excites high-energy states of the solid Ne.

\section{Results and discussion}

Figure 1, shows ECR signal intensity, $A$, open circles, and gas pressure in the microwave cavity $p_{\text {res}}$, open triangles, for the $4.2 \mathrm{~K}$ sample versus pressure $p$ measured at the warm end of the tube supplying the gaseous $D_{2}$ to the

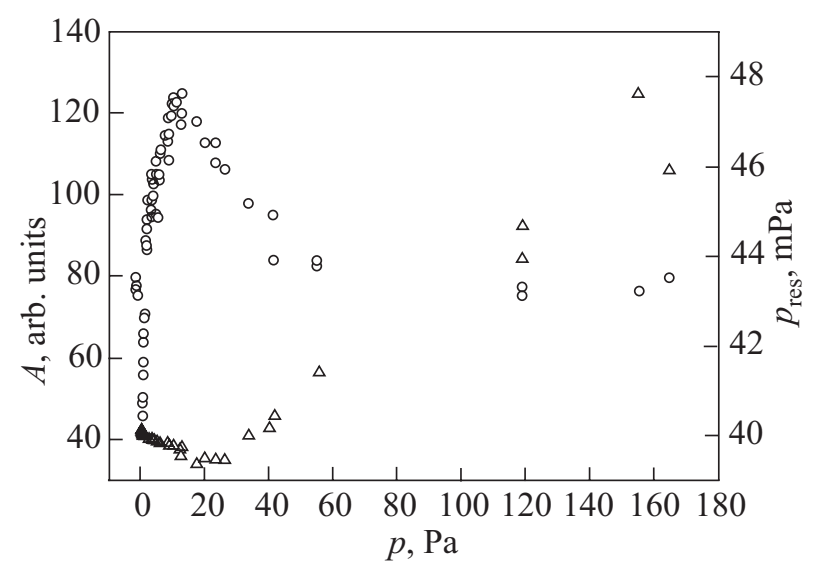

Fig. 1. ECR signal intensity $A$ (open circles), and gas pressure in the microwave cavity, $p_{\text {res}}$, (open triangles), for the $4.2 \mathrm{~K}$ sample versus pressure $p$ measured at the warm end of the tube supplying the gaseous $\mathrm{D}_{2}$ to the substrate. The pressure is proportional to the rate of the $\mathrm{D}_{2}$ impurity gas flow. substrate. The pressure is proportional to the rate of the $\mathrm{D}_{2}$ impurity gas flow.

This warm end was attached to a needle valve which governed $p$ and, hence the He flow. The flow might also be assessed by the gas amount consumed from the storage container. The rate of an impurity gas flow $q$ depends linearly on $p$ [2]. Intensity data are obtained based on the amplitude and width of the ECR resonance. Some excess broadening was recorded at large deuterium flows. The intensity is corrected to the broadening as being proportional to the squared peak-to-peak ECR derivative width. The experimental data on the broadening may bring information on the collisions of the emitted electrons in the near vicinity of the sample surface. Indeed, an assessment of the Larmor radius suggests that the electron is localized very close to the surface which it has escaped. For the kinetic energy of the emitted electrons of some hundred Kelvin [4], a Larmor radius is several micrometer for the electrons in magnetic field of $0.33 \mathrm{~T}$. Generally, the ECR line broadening may be considered as due to the electron-neutrals collisions, electron-electron collisions, and nonuniformity of the magnetic field across the cavity $[3,4]$. The above excess broadening may be considered as due to the either collisions between the free electrons and neutral $\mathrm{Ne}$ atoms and $\mathrm{D}_{2}$ molecules or scattering of the electrons by surface roughness. The latter process is probable to contribute noticeably to the width because of the small Larmor radius of the ejected electron and extreme roughness of the $\mathrm{Ne}$ quench condensed film. As a result, the increase in the peak-to-peak width $\Delta H$ upon increasing pressure $p_{\text {res }}$, in the microwave cavity is observed for the ECR adsorption line. The similar effect was found out previously in the $\mathrm{Ne}-\mathrm{He}$ experiments [1,2]. It was observed with far lager range of the $p_{\text {res }}$ variation which makes it possible to plot $\Delta H$ against $p_{\text {res }}$, Fig. 2.

The excess width is proportional to the gas pressure as expected for the ECR adsorption of free electrons in partially ionized gases [20]. Thus, the present $\mathrm{Ne}-\mathrm{D}_{2}$ experiment as well as the previous $\mathrm{Ne}-\mathrm{He}$ measurements substantiate that the electron-neutrals collisions may contribute markedly to the ECR linewidth in our experiments. This is probably due to the fact that most of the free electrons are orbiting in the external magnetic field close to the sample surface, thus, interacting with both the neutral particles at large concentration and surface roughness. Earlier [4], al- 


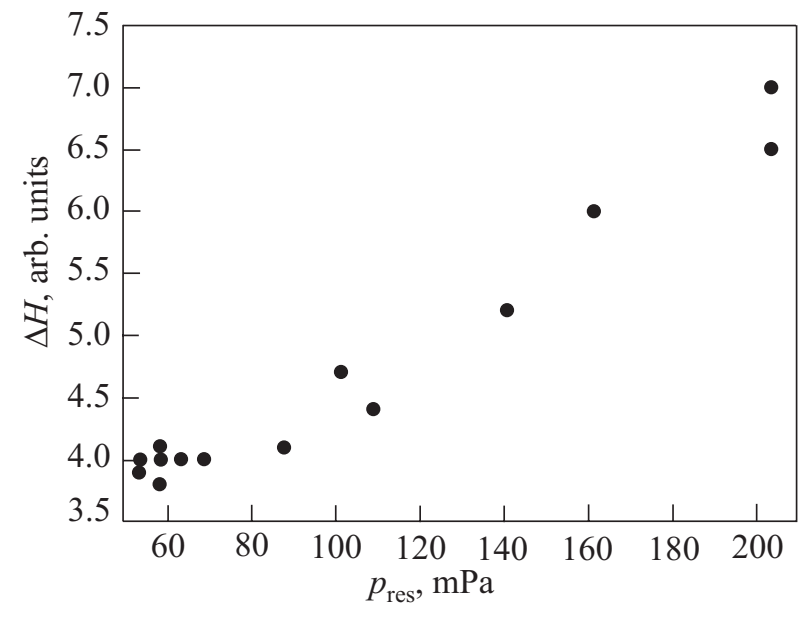

Fig. 2. Linewidth of the ECR adsorption $\Delta H$ for the $4.2 \mathrm{~K}$ sample versus pressure $p$ measured at the warm end of the tube supplying the gaseous He to the substrate. The pressure is proportional to the rate of the He impurity gas flow.

most the whole ECR broadening was attributed to the electron-electron collisions.

Figure 1 suggests the nonmonotonic dependence of $A$ on $p$ : increase in the ECR signal intensity at relatively small $\mathrm{D}_{2}$ impurity concentration in solid $\mathrm{Ne}$, while decrease in the intensity at large impurity concentration. Thus, the effect of the $\mathrm{D}_{2}$ impurity is in marked contrast with effects which $\mathrm{He}$ and $\mathrm{CH}_{4}$ impurities have on the photoelectron emission from solid $\mathrm{Ne}$. Both, $\mathrm{He}$ and $\mathrm{CH}_{4}$, dopants decrease significantly the free electron emission from the solid $\mathrm{Ne}[1,2,5]$. An analysis of the experimental data, including fitting of the data, showed $[1,2,5]$ that the surface impurities played the major role in emission quenching, while the bulk impurities have negligible effect on the emission compared to the surface ones. Furthermore, the discussions of the models of the active surface sites suggested that the flat surface is inactive in the photoemission which occurs, most probably, through the low-coordinated surface sites. Electrons are found to be delocalized in the bulk solid Ar [6,21] and $\mathrm{Ne}$ [21]. In nominally pure matrices of light gases, the electrons can be trapped only by such lattice defects as vacancies and vacancy clusters [6] due to negative electron affinities of these matrices. Some of the electrons generated in the conduction band by UV irradiation and traveling inside the solid can eventually reach the surface. It is known that charges (electrons and holes) can be trapped at topological features, such as steps on the surfaces of insulating materials. The irradiation enables the trapped surface electrons to escape rapidly into vacuum from these shallow traps. Impurities, $\mathrm{He}$ and $\mathrm{CH}_{4}$, adsorbed at those cites, turned out to be effective traps in solid Ne, thus, not allowing the electrons to escape into the vacuum. Surprisingly, this occurs despite the affinities of different signs of $\mathrm{He}$ and $\mathrm{CH}_{4}$.
Effects of $\mathrm{He}$ and $\mathrm{CH}_{4}$ doping on the photoelectron emission from solid $\mathrm{Ne}[1,2,5]$ are related to radiationinduced charging in polycrystalline insulating materials. Recently, it was demonstrated by first-principles calculations that the surface trapping of electrons occurs even in negative-electron-affinity materials $[22,23]$. By examining insulators like $\mathrm{MgO}, \mathrm{LiF}$ and $\mathrm{NaCl}$, the authors showed that low-coordinated sites and grain boundaries may act as traps for conduction-band electrons. The calculations are substantiated by experimental findings on UV desorption of neutral $\mathrm{Mg}$ and $\mathrm{O}$ atoms from particular $\mathrm{MgO}$ sites [24,25]. The proposed mechanism of desorption involves localization of excitons as well as electrons and holes at three-coordinated surface sites-corners and kinks.

The role of low-coordinated surface sites becomes more convincing when one relates to the different results for hampering the electron emission by $\mathrm{He}$ adsorption observed for the samples at $4.2 \mathrm{~K}$ and $1.6 \mathrm{~K}[1,2]$. The impurity He gas flow has no effect on the electron yield from the Ne samples at $4.2 \mathrm{~K}$ while hampers the yield by an order of magnitude for the samples at $1.6 \mathrm{~K}$. This is attributable to the small binding energy of a He atom on the Ne substrate. The van der Waals constant $C_{3}$ the well depth $D$ and the binding energy $E_{b}$ of the ${ }^{4} \mathrm{He}$ - flat neon substrate interaction potential are not known precisely from the measurement. Realistic estimation of this value may be obtained in the harmonic approximation [26]:

$$
E_{b}=-D+\frac{\hbar \omega_{1}}{2}
$$

where $\omega_{1}$ is the zero-point vibration frequency obtained from the near universality of physisorption potentials determined from experiments on inelastic He atom scattering on various surfaces [27]:

$$
\omega_{1}=\sqrt{\frac{k}{m}} D^{\frac{5}{6}} C_{3}^{-\frac{1}{3}} .
$$

Here $k=21$ and $m$ is the ${ }^{4} \mathrm{He}$ mass, $D=60 \mathrm{~K}, C_{3}=$ $=163 \mathrm{~K} \cdot \AA$, from Ref. 27 and references therein. As a result, we calculated the binding energy from Eqs. (2) and (3) to be $44 \mathrm{~K}$, which is in agreement with $E_{b}=37 \mathrm{~K}$ for ${ }^{3} \mathrm{He}$ on the same substrate, see [28] and references therein. He atoms, which are initially adsorbed on a terrace, can then either diffuse on the terrace to the step edges and be trapped there or be desorbed from the Ne surface. The time for hopping between terrace sites:

$$
\tau_{h}=\tau_{0} \exp \left(\frac{E_{b}}{2 T}\right) .
$$

The time of adsorption on a terrace:

$$
\tau_{a}=\tau_{0} \exp \left(\frac{E_{b}}{T}\right) .
$$

Here $\tau_{0}=10^{-13} \mathrm{~s}$. 
If we take the terrace linear dimension $L$ to be of the order of the size of a crystallite, than, roughly, $L \sim 100 \mathrm{~nm}$ [29]. Based on this estimation and the Ne lattice constant (the hopping length), $a=0.3153 \mathrm{~nm}$, we obtain number of diffusion hops which a $\mathrm{He}$ atom needs to reach the step edge via random walk:

$$
N=\left(\frac{L}{a}\right)^{2} .
$$

Equations (4) and (6) give us the diffusion time:

$$
\tau_{d}=\tau_{h} N
$$

The ratio $\tau_{a} / \tau_{d}$ gives an estimate of the chance that $\mathrm{He}$ atom will be trapped at the step edge. Let us take $E_{b}=$ $=37 \mathrm{~K}$. Then the ratio $\tau_{a} / \tau_{d}$ will be $8 \cdot 10^{-4}$ and 1 , for $4.2 \mathrm{~K}$ and $1.5 \mathrm{~K}$ samples, respectively. Thus, a $\mathrm{He}$ atom initially adsorbed on the terrace is likely to be trapped at the step edge of the $1.5 \mathrm{~K}$ sample, while would be desorbed from the $4.2 \mathrm{~K}$ terrace. On the other hand, cryopumping of small He flows by $\mathrm{Ne}$ takes place in the $4.2 \mathrm{~K}$ samples [2], which is evidenced from nonlinear $p\left(p_{\mathrm{He}}\right)$ dependence, where $p_{\mathrm{He}}$ is the helium pressure in the cavity. This may be achieved through the sequence of adsorption-desorption processes which a He atom experiences on the surface of highly porous Ne sample until the atom reaches a site with high binding energy or escape into the vacuum. With increasing He flow, these sites are "saturated" and $p\left(p_{\mathrm{He}}\right)$ dependence becomes linear. Based on the accommodation times, one can assume that the above "special" sites (sites with a large binding energy) are adatom positions with 8 or 9 neighbors [29] rather than the step edges with 5 neighbor $\mathrm{Ne}$ atoms [29]. Indeed, for the $4.2 \mathrm{~K}$ sample, the time of adsorption on the step edge will be:

$$
\tau_{\text {edge }}=\tau_{0} \exp \left(\frac{E_{b} \frac{5}{3}}{T}\right) \approx 2.4 \cdot 10^{-7} \mathrm{~s},
$$

which is much below the time of the monolayer formation, $\tau_{\text {mono }}=0.02 \mathrm{~s}$, estimated from the rate of the Ne flow. Hence, the He atom will be desorbed from the step and not trapped there. On the other hand, the sites with 8 and $9 \mathrm{Ne}$ neighboring atoms yield $\tau_{\text {edge }}$ of $1.6 \cdot 10^{-3} \mathrm{~s}$ and $0.03 \mathrm{c}$ respectively, which is much closer to $\tau_{\text {mono. Vacuum cryo- }}$ condensates of van-der-Waals (vdW) gases, solid Ne including, were shown earlier [30] to be effective sorbents for helium with physical mechanism of adsorption. The connection of adsorption values was related to the structural and thermophysical parameters of cryocondensates.

Thus, the analysis of the accommodation times substantiates the suggestion that photoelectrons escape the sample from regions where two Ne planes cross, probably, step edges.
The $\mathrm{Ne}-\mathrm{He}$ and $\mathrm{Ne}-\mathrm{CH}_{4}$ experiments discovered that the ECR amplitude started to decrease at comparatively low gas impurity flows related to the $\mathrm{p}$ values of: $0.2 \mathrm{~Pa}$, in $\mathrm{Ne}-\mathrm{He} 1.6 \mathrm{~K}$ runs, $0.04 \mathrm{~Pa}$, in $\mathrm{Ne}-\mathrm{CH}_{4} 4.2 \mathrm{~K}$ runs, and $0.3 \mathrm{~Pa}$, in $\mathrm{Ne}-\mathrm{CH}_{4} 1.6 \mathrm{~K}$ runs. In any event, the photoemission yield dropped by several times before the pressure, $p$, at the warm end of the tube supplying dopant reached a value of $2 \mathrm{~Pa}$. Given the dynamic viscosity coefficients at $273 \mathrm{~K}$ of: $19 \mu \mathrm{Pa} \cdot \mathrm{s} \mathrm{He}, 11 \mu \mathrm{Pa} \cdot \mathrm{s} \mathrm{CH}_{4}$, and 12.5 $\mu \mathrm{Pa} \cdot \mathrm{s} \mathrm{D}_{2}$ (assessed from $8.8 \mu \mathrm{Pa} \cdot \mathrm{s} \mathrm{H}_{2}$ ), the flow rates of the above gases should be rather close at close $p$ quantities. Thus, Fig. 1 suggests that no quenching of the photoelectron emission from $\mathrm{Ne}$ surface low-coordinated sites was observed in $\mathrm{D}_{2}$ doping experiments. On the contrary, the ECR signal amplitude was found to increase markedly at pressures lower than about $10 \mathrm{~Pa}$. Some hint to explain the increase of the emission may be obtained from the observations as follows. A part of the gas supplying line between the closed needle and a shut valves $\left(100 \mathrm{~cm}^{3}\right.$ approximate volume) was filled with a dopant gas up to several hundreds $\mathrm{Pa}$. Then the shut valve was opened and the pulse gas flow was supplied to the solid Ne sample being under the action of the open $\mathrm{Ne}$ gas discharge. Immediately, the ECR signal multiplied several times its amplitude, regardless of the type of dopant gas used. Figure 3 presents ECR signals recorded both before and some minutes after the shut valve was opened. The effect of the abrupt amplitude rise was observed not only with the use of $\mathrm{D}_{2}, \mathrm{O}_{2}$, and $\mathrm{Ne}$ gaseous dopants, Fig. 3, but with $\mathrm{He}$ and $\mathrm{CH}_{4}$ ones as well.

With the gradual evacuation of the $100 \mathrm{~cm}^{3}$ volume through the gas supplied matrix line, the ECR signal grew smaller and reached amplitude close to the one recorded

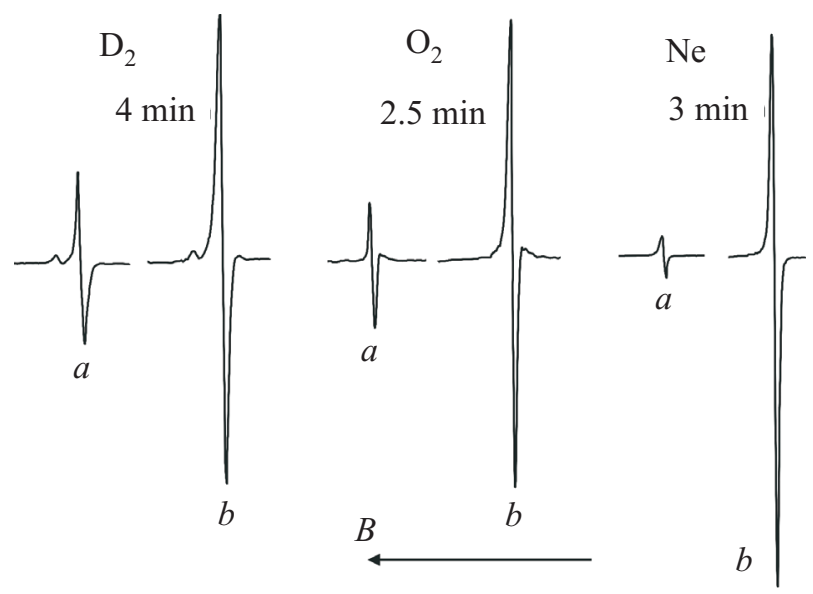

Fig. 3. Photoelectron emission from solid Ne. The figure demonstrates abrupt increase in the ECR adsorption intensity when the pulse gas flow is supplied to the substrate through the matrix channel: before the shut valve is opened $(a)$; certain minutes after the shut valve was opened $(b)$. The figure shows results obtained with the use of molecular deuterium, oxygen, and atomic neon. The liquid helium temperature in the cryostat is $4.2 \mathrm{~K}$. 
before the shut valve was opened. The time change of the intensity is seen from Fig. 4. The clue to the explanation of this step like growth of the electron emission upon the action of the pulse gas flow lies most probably in the finding of shallow electron traps in the rare gas solids (RGS) possessing negative electron affinity. Thus, considerable concentration of electrons trapped in solid neon matrix was reported by Frankowski et al. [31] and Savchenko et al. [32]. The authors measured thermally stimulated exoelectron emission (TSEE) from solid neon layers formed by depositing the gas on a cold substrate with concurrent electron irradiation.

The electron emission from pre-irradiated samples was detected in two types of experiments: upon gradual heating of the sample at a steady rate and in a series of discrete, 1 $\mathrm{K}$ heating steps. The trapped electrons were shown to be localized not in well-defined traps of uniform depth and with identical activation energy, but in a nearly continuous distribution of traps with varying depths. Testing whether the observed electrons are extracted from the entire volume of the sample or only from the surface layers, the authors came to the conclusion that most of the obtained exoelectron current originates in the top 10-20 $\mu \mathrm{m}$ of the sample. Shallow electron traps were also observed in solid Ar, another rare gas matrix with negative electron affinity $[33,34]$. In the first report of TSEE from solid Ar [33], one of the TSEE peaks, at $12 \mathrm{~K}$, was related to surface traps and traps at inner interfaces in the sample. Even shallower TSEE peak was observed at $9 \mathrm{~K}$. It is worth noting two peculiarities of the TSEE yield the authors stressed for Ne and Ar films. First, the concentration of trapped electrons was much higher in quench-condensed unannealed films. This was attributed to the higher number of electron traps in unannealed films [34]. The TSEE yield from films grown by layered deposition under electron beam irradia-

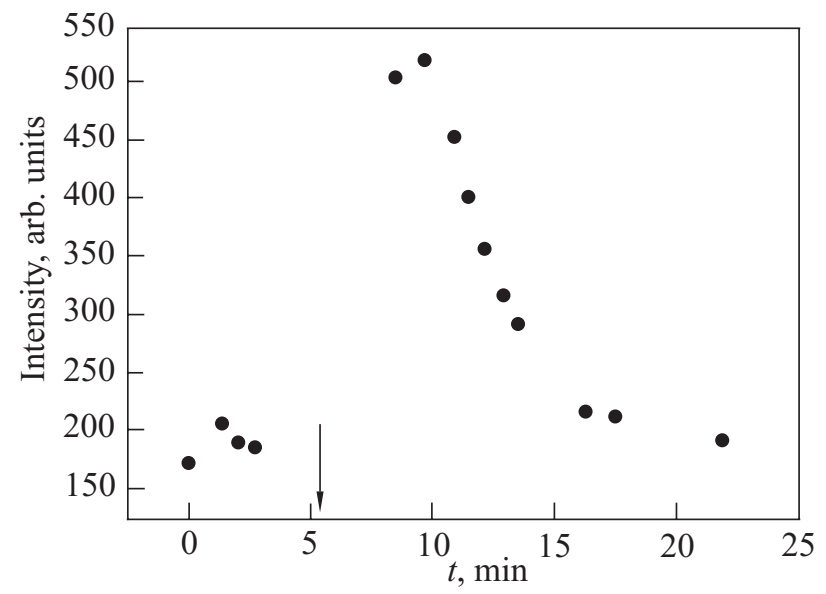

Fig. 4. Time variation of the ECR adsorption intensity after the shut valve was opened thus supplying the pulse flow of gaseous $\mathrm{D}_{2}$ to the substrate. The arrow indicates the moment when the valve was opened. tion exceeded that detected from the samples irradiated after deposition [31,34]. Thus, one may expect that, in our experiments where the $\mathrm{Ne}$ gas is quench-deposited being under the action of the excitation radiation, the conditions are favorable to observe large electron emission from the sample.

We believe that our experiments with pulse gas flows give additional support to the previous finding of the formation of shallow electron traps on the Ne surface. In that case, the heat delivered by any gas condensing on the sample surface promotes the electrons from the traps into the vacuum. In the previous ECR study [4] of the electron emission from solid $\mathrm{Ne}$, an effect of the sample temperature on the emission was observed and measured. The temperature was varied in the range from 4.2 through 21 $\mathrm{K}$. We recorded an emission drop by the order of magnitude with the sample temperature increasing from about 7 to $12 \mathrm{~K}$. The discrepancy between the temperature effect on the emission reported in the present and previous [4] study lies, supposedly, in the fact that the rise of the substrate temperature [4] brings about the temperature increase of the entire bulk of the Ne film, while adsorption of the large gaseous flow causes primarily heating of the surface with the result of the temperature gradient through the sample thickness. The conduction electrons appear in the bulk of the solid Ne condensed from discharge and subjected to the action of the excitation radiation. Some electrons reach the surface and are ejected into the vacuum, while some are trapped in the defect sites. Two processes may be supposed to give rise to the decrease of the emission yield obtained in Ref 4 . The first one may be that the increasing Ne bulk temperature would give rise to a drop in the efficiency of the formation of electrons in the conduction band. The second one is based on an assumption that the probability of self-trapping of electrons decreases with increasing temperature, while their mobility increases, and hence the rate of formation of luminescence centers increases sharply during recombination [35] manifesting the lost conduction electrons. On the contrary, a moderate and inhomogeneous heating of the sample surface by the pulse gaseous flow frees up electrons from the shallow traps influencing no efficiency of the formation of the electrons in the conduction band in the bulk.

In contrast to the $\mathrm{He}$ and $\mathrm{CH}_{4}$ dopants, the study with $\mathrm{D}_{2}$ dopant produced no reliable effect of the sample temperature changing in the range $1.5-4.2 \mathrm{~K}$ on the electron emission. Most probably, this finding is in line with the inference about the nearly continuous distribution of traps with varying depths at the Ne surface [31].

As was noted above, the $\mathrm{D}_{2}$ doping effect differs significantly from that for $\mathrm{He}$ and $\mathrm{CH}_{4}$ impurities in that the $\mathrm{D}_{2}$ impurity turns out to be capable of increasing the electron emission from the matrix. Because of the very similar Lennard Jones potentials of the $\mathrm{D}_{2}$ and Ne solids, the $\mathrm{D}_{2}$ molecules may be considered a near isotopic impurity. Therefore, 
the deuterium adsorbate has no negative effect on the emission of electrons from the steps on the Ne surface. Furthermore, mobile $\mathrm{D}_{2}$ atoms diffusing across the Ne surface may be trapped at the terrace defect sites, thus "healing" the defect structure. As a consequence, lesser quantity of the terrace surface traps is available for the electrons which eventually appear on the steps which are active to the electron emission. The above mechanism implies noticeable solubility of a dopant in the solid Ne. The solubility of $\mathrm{He}$ and $\mathrm{CH}_{4}$ in $\mathrm{Ne}$ is close to zero, as opposed to the large fraction of the $\mathrm{D}_{2}$ component found in the $\mathrm{D}_{2}-\mathrm{Ne}$ solid mixtures.

Let $A(p)$ be the ECR signal intensity depending on the $p$ which is proportional to the flow rate of the gaseous dopant. The fitting of $A(p)$ may justify the assumption about the role of the surface $\mathrm{D}_{2}$ atoms and provide valuable information on the process of the photoelectron emission. It is reasonable to take the number of electrons escaped the surface traps due to the "healing" the defect structure by adsorbed $\mathrm{D}_{2}$ molecules as proportional to the $\mathrm{D}_{2}$ concentration $n$. It is also reasonable to assume that this concentration shows a maximum $n_{\max }$, at a solid solubility limit for $\mathrm{D}_{2}$ in quench condensed Ne. Our experiments evidence occurrence of the limit, at large deuterium flows, through sudden bursts of the pressure which follow partial sample decomposition. In that case one could take the increment of $n$ as

$$
\Delta n \sim\left(n_{\max }-n\right)^{\gamma} \cdot \Delta p
$$

where $\Delta p$ is proportional to the increment of the deuterium gaseous flow rate. Our fitting procedure showed a reliable exponent $\gamma$ to be 2 .

Hence, the $A(p)$ dependence, which accounts also for the emission taking place from the Ne surface regions free from adsorbed $\mathrm{D}_{2}$ molecules, can be written in the form:

$$
A(p)=a_{1}+\frac{b_{1}}{1+c_{1} p}
$$

with constants $a_{1}, b_{1}$, and $c_{1}$ to be obtained in the fitting procedure.

Curve 1 (Fig. 5) plotted using Eq. (7) matches well experimental results at small $\mathrm{D}_{2}$ flows. The decrease in the intensity with increasing deuterium flow above approximately $10 \mathrm{~Pa}$ may be explained based on the assumption that solid $\mathrm{D}_{2}$ microcrystals are formed in the sample. Previously [5], perceptible effect of the $\mathrm{CH}_{4}$ microcrystal formation on the electron emission from the Ne sample was found at the dopant pressure exceeding $p \approx 2 \mathrm{~Pa}$. The larger is the deuterium flow the lesser is the free Ne surface $S_{\text {free }}$ ejecting the electrons into the vacuum. Based on the dopant crystal concentration proportional to $p^{d_{3}} /\left(1+k_{2} p^{d_{3}}\right)$ with $d_{3}=2$ [5], we came to the conclusion that the $\mathrm{Ne}$ crystal concentration would be proportional to $1 /\left(1+k_{2} p^{d_{3}}\right)$. Then we obtain the fitting expression:

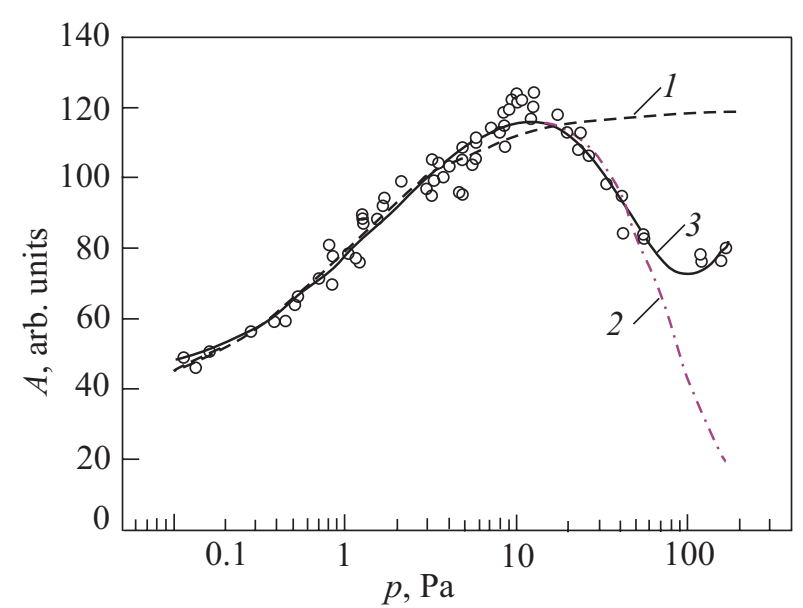

Fig. 5. The intensity of the ECR signal $A$ for the $4.2 \mathrm{~K}$ neon sample versus pressure $p$ measured at the warm end of the tube supplying the gaseous $\mathrm{D}_{2}$ to the substrate. Open circles - experimental data; fitting curves: a curve which fits the experimental data obtained at small $\mathrm{D}_{2}$ flow rates; it was calculated in the assumption that the surface $\mathrm{D}_{2}$ molecules "healed" the defect structure of the Ne surface (1); a curve fits experimental data obtained at small and moderate $\mathrm{D}_{2}$ flow rates; the curve accounts for the "healing" effect (like curve 1) and allows also for the effect that the concentration of the Ne microcrystals decreases with increasing the $\mathrm{D}_{2}$ flow rate (2); a curve which fits the experimental data in the whole pressure region; besides the effect which adsorbed $\mathrm{D}_{2}$ molecules have on the emission from the Ne surface, the curve accounts for the formation of $\mathrm{D}_{2}$ microcrystals and heating of the sample; the heating results in the thermally induced emission of electrons from shallow traps (3).

$$
A(p)=\left(a_{1}+\frac{b_{1}}{1+c_{1} p}\right) \cdot \frac{1}{1+c_{2} p^{2}} .
$$

The fitted curve 2 plotted with the use of Eq. (8) is in good agreement with the experimental data obtained at not too large values of $p$. One could suppose that the large dopant flows cause some heating of the sample with the result of the thermally induced emission of electrons from shallow traps. The surface temperature of the sample is proportional to $p$. Eventually we obtained the fitting expression which accounts also for the solid deuterium microcrystal formation which contributes to the emission with a term proportional to $p^{2} /\left(1+d_{1} p^{2}\right)$ :

$$
A(p)=\left(a_{1}+\frac{b_{1}}{1+c_{1} p}\right) \frac{1}{1+d_{1} p^{2}}+a_{2} p+\frac{b_{3} p^{2}}{1+d_{1} p^{2}} .
$$

An agreement between the theoretical model and experiment, curve 3 (Fig. 5), may be considered as rather good. At small $\mathrm{D}_{2}$ flows, the curves 2 and 3 overlap thus being undistinguishable.

Constants $a_{1}, b_{1}$, and $c_{1}$ obtained in fitting procedure (9) makes it possible to get a crude estimate of the solid 
solubility limit for $\mathrm{D}_{2}$ in quench condensed $\mathrm{Ne}$. Based on the consumption of gaseous $\mathrm{Ne}$ and $\mathrm{D}_{2}$ from the storage volumes during experimental runs and a geometry of the gas deposition arrangement in the microwave cavity, we assess that, at $p \approx 10 \mathrm{~Pa}$, the $\mathrm{D}_{2}$ flow rate to the substrate is nearly equal to the $\mathrm{Ne}$ flow rate, thus, making 0.28 $\mathrm{mmol} / \mathrm{h}$. While $\mathrm{H}_{2}$ crystals are stable enough that $\mathrm{Ne}$ is not soluble in $\mathrm{H}_{2}$ to any significant extent, $\mathrm{H}_{2}$ is soluble in $\mathrm{Ne}$, however $[36,37]$. In that case, the model we used of the physical mixture of Ne microcrystals doped with $\mathrm{D}_{2}$ molecules and pure $\mathrm{D}_{2}$ crystallites seems to be reliable. The initial ECR signal intensity, $A(0)$, is calculated as $A(0)=$ $=a_{1}+b_{1}$ and relates to the emission from pure Ne crystallites. The coefficient $a_{1}$ presents an asymptotic value of the intensity $A(\infty)=a_{1}$ being proportional to the solid solubility limit for $\mathrm{D}_{2}$ in quench condensed Ne. Next we assume that $\mathrm{D}_{2}$ concentration of $1 \%$ is completely dissolved in $\mathrm{Ne}$. Indeed, $\mathrm{H}_{2}$ was previously found to be dissolved in quench condensed $\mathrm{Ne}$ with $\mathrm{H}_{2}$ concentrations up to $40 \%$ [36] at $5 \mathrm{~K}$. The $\mathrm{D}_{2}$ concentration of $1 \%$ in Ne corresponds to $p \approx$ $\approx 0.1 \mathrm{~Pa}$. Then, because of the assumed proportionality between the $\mathrm{D}_{2}$ concentration and excess emission of electrons, solid solubility limit may be obtained as

$$
\begin{gathered}
\frac{a_{1}-\left(a_{1}+b_{1}\right)}{A(0.1)-\left(a_{1}+b_{1}\right)} 0.01= \\
=\frac{127.8894-(127.8894+(-85.0025))}{48.2721-(127.8894+(-85.0025))}=0.158 .
\end{gathered}
$$

Thus, the crude estimate of the solid solubility limit of $\mathrm{D}_{2}$ in quench-condensed $\mathrm{Ne}$ is $16 \%$. In samples rich of $\mathrm{Ne}$, appearance of the hcp phase with lattice volume close to that of the solid $\mathrm{D}_{2}$ was observed only with concentrations over $50 \%$ of $\mathrm{D}_{2}$ in gaseous $\mathrm{Ne}-\mathrm{D}_{2}$ mixture [37]. Lesser concentrations brought about two solid structures, fcc and hcp phases, with lattice volumes close to that of the pure Ne. A monophase fcc mixture was found for deuterium concentrations below 5\%. Belan et al. [38] performed xray investigations of vacuum condensates of the binary mixtures $\mathrm{Ne}-n \mathrm{D}_{2}$ at temperatures from $6 \mathrm{~K}$ to the melting point of the solutions. In the intermediate concentration range of $\mathrm{D}_{2}$ in $\mathrm{Ne}$, they observed a metastable hexagonal $\mathrm{hcp}_{2}$ phase with volume close to the volume of the neon lattice together with the equilibrium hcp $\mathrm{p}_{1}$ and fcc phases of solid deuterium and neon, respectively. It turned out that as the deuterium concentration increases above 4.5 mole $\% \mathrm{D}_{2}$ completely dissolves in the metastable hcp 2 phase. In addition, the amount of the latter phase increases linearly and that of the fcc phase decreases with increasing concentration of $n \mathrm{D}_{2}$ molecules. For deuterium concentrations above $50 \mathrm{~mole} \%$, the amount of the fcc phase in the samples with condensation on a substrate at temperature $6 \mathrm{~K}$ is so small that within the sensitivity of the experiment only two hexagonal phases - hcp ${ }_{1}$ and hcp 2 - were formed when the samples were condensed at low temperatures. The authors [38] referred to the estimates of the highest $\mathrm{H}_{2}$ concentrations in solid Ne up to $10 \%$.

The model we use, see Eq. (9), implies no abrupt appearance of the pure $\mathrm{D}_{2}$ hcp phase with increasing deuterium concentration above $50 \%$, the "threshold" concentration. However, the equation shows rather fast rise of the $\mathrm{D}_{2}$ microcrystal concentration with increasing $p$. A very rough assessment of the $\mathrm{D}_{2}$ "threshold" concentration may be obtained from our model if we take a position of the maximum of the derivative of $A_{3}(p)=b_{3} p^{2} /\left(1+d_{1} p^{2}\right)$ function as indicative of such a concentration. With $d_{1}=$ $=0.00047$, obtained in fitting procedure, we found out $p=$ $=26 \mathrm{~Pa}$ at the maximum derivative of $A_{3}(p)$ which makes the "threshold" concentration to be $70 \%$.

Thus, the above estimate of the solubility limit is in general agreement with the previous results on the structure of the $\mathrm{Ne}-\mathrm{D}_{2}$ solid solutions.

Let us consider a maximum temperature gradient through the sample thickness which happens because of the heat of condensation loaded on the sample surface at maximum deuterium flows. To give a hint about the thickness of the sample film let us consider the largest magnitude of the gaseous $\mathrm{D}_{2}$ flow of $4.4 \mathrm{mmol} / \mathrm{h}$ when the sample is generally the deuterium solid. Such a flow provided for an hour makes 4.4 mole of solid $\mathrm{D}_{2}$ at the substrate surface, i.e., the bottom of the quartz finger. The bottom area is approximately $S=1 \mathrm{~cm}^{2}$. Given the solid $n-\mathrm{D}_{2}$ lattice volume of $20 \mathrm{~cm}^{3} / \mathrm{mol}$ [39], the film thickness would be about $1 \mathrm{~mm}$. The solid $\mathrm{D}_{2}$ thermal conductivity at $4 \mathrm{~K}$ and heat of the phase transfer (when the temperature is lowered from 80 to $4 \mathrm{~K}$ ) are $\approx 100 \mathrm{~W} \cdot \mathrm{m}^{-1} \cdot \mathrm{K}^{-1}$ and $3.483 \mathrm{~kJ} / \mathrm{mol}$, respectively [29]. Then, the temperature gradient would be $\Delta T \approx 0.4 \mathrm{mK}$ for the $1 \mathrm{~mm}$ thick film. The heat resistance of the quartz finger bottom is expected to give larger contribution to the surface temperature of the solidified gas layer. Given the bottom thickness of $d=0.5 \mathrm{~mm}$, we estimate the thermal flow: $P=\frac{S}{d} \int_{4}^{6} \lambda d T=42 \mathrm{~mW}$. Here, $\int_{4}^{6} \lambda d T=0.211 \mathrm{~W} / \mathrm{m}$ is the integral thermal conductivity of the fused silica [29]. The maximum thermal flow during gas condensation was assessed to be $4.3 \mathrm{~mW}$ suggesting, thus, the temperature gradient through the bottom thickness of $0.2 \mathrm{~K}$. With so moderate an averaged surface heating at hand, we believe that the thermally activated electron emission comes from some surface regions with high enough temperatures which happen to exist because of the nonuniform temperature distribution across the sample growing from the gaseous $\mathrm{Ne}-\mathrm{D}_{2}$ mixture. One could suppose that the overheating of these surface regions is due to the lattice relaxation of the quench condensed $\mathrm{Ne}-\mathrm{D}_{2}$ mixture. As was mentioned in the present paper, the release of a large amount of heat during the matrix structure relaxation is evident through the pressure bursts which occur in the cavity at times during growth of the sample with large impurity concentration. 
Figure 1 shows increase in $p_{\text {res }}$ of about $8 \mathrm{mPa}$ at larger deuterium flows thus suggesting partial lost of the gaseous deuterium supplied onto the substrate. This means that a part of the dopant was pumped by the pumping facilities of the set up through an annular gap between the quartz finger and a microwave cavity cover. The lost however is estimated to be negligible, which is evidenced from the fact that only $1 \mathrm{~Pa}$ increase in the pressure $p$ of the noncondensing gaseous component causes rise in pres of $22 \mathrm{mPa}$, see Fig. 2 in Ref. 2. If one allowed for the above $8 \mathrm{mPa}$ increase in $p_{\text {res }}$ the correction to $p$ would be about $0.36 \mathrm{~Pa}$, i.e., insignificant.

\section{Conclusions}

The effect of the $\mathrm{D}_{2}$ doping on the electron photoemission from solid $\mathrm{Ne}$ was studied at the sample temperatures from $1.5 \mathrm{~K}$ to $4.2 \mathrm{~K}$. As opposed to the experiments with $\mathrm{He}$ and $\mathrm{CH}_{4}$ impurities the deuterium one yields nonmonotonic dependence of the emission on the impurity concentration. What is more surprising is that the emission shows increase with increase in the dopant concentration. Possibly the electron emission peculiarities of the $\mathrm{D}_{2}$ doped $\mathrm{Ne}$ relate to the exceptional properties which the neon-hydrogen mixture is believed to have. The molecular volume of the neon is small enough relative to the hydrogen one that it falls in the proper range of relative species sizes to form a Laves phase crystal of the form $\mathrm{H}_{2}(\mathrm{Ne})_{2}$ or $\mathrm{D}_{2}(\mathrm{Ne})_{2}$ similar to the vdW compounds formed at high pressure. $\mathrm{D}_{2}$ can also be considered an isotopic impurity in $\mathrm{Ne}$ with very similar Lennard-Jones potential parameters: $\sigma=2.96 \AA, \varepsilon=36.7 \mathrm{~K}$ and $\sigma=2.79 \AA, \varepsilon=36.7 \mathrm{~K}$, for the $\mathrm{D}_{2}$ and $\mathrm{Ne}$ solids, respectively [39]. The difference occurs mostly as a result of the difference in the zero point energies. The degree of quantum effects is quantified by the de Boer quantum parameter which is 0.193 , for $\mathrm{D}_{2}$, and 0.0918 , for $\mathrm{Ne}$ [39]. The deuterium is also known to be soluble in rather large concentrations in solid $\mathrm{Ne}$ when deposited from the gas phase $[37,38]$. The tentative explanation of the electron emission increase with increase in the $\mathrm{D}_{2}$ concentration is that lesser quantity of the terrace traps are available for the electrons which eventually appear at the low-coordinated step sites active with respect to the electron emission. At concentrations smaller than $4.5 \%$, the mobile $\mathrm{D}_{2}$ molecules would occupy the surface traps thus "healing" the defect structure of the Ne surface. At larger concentrations, new hcp 2 phase appears [38] contributing to the electron emission. Very crude estimate gives the $\mathrm{D}_{2}$ content of the phase of about $16 \%$. With increase of the $\mathrm{D}_{2}$ concentration in the gaseous mixture supplied to the low-temperature substrate, the metastable hcp 2 phase substitutes the stable fcc phase. The concentration of the latter one, thus, gradually decreases [38]. One could suppose, that the $\mathrm{hcp}_{2}$ is a less defective structure than the fcc because of the large amount of light $\mathrm{D}_{2}$ component. As a result, one could expect larger free electron yield from the metastable phase manifesting in the increase of the ECR signal intensity. The model we used accounted also for both the formation of deuterium microcrystals and thermally induced electron emission at large gaseous flow rates onto the sample surface.

The VUV photoinduced electron emission from rare gas solids (RGS) was found to be orders of magnitude larger than that from molecular gas solids $[3,4]$. This finding correlates with other studies on the electron emission from rare gas solids. Baragiola et al. [40] studied electron emission from solid $\mathrm{Ar}, \mathrm{Kr}$, and $\mathrm{Xe}$ films induced by impact of $10-100 \mathrm{keV}$ protons of normal incidence. The observed electron yields turned out to be huge - hundreds of electrons per incident ion: higher per amount of electronic energy deposited, than for any other material studied. The secondary electron yield was measured for solid Xe films excited by $1-10 \mathrm{keV}$ x-rays [41]. The authors observed high electron yields and demonstrated that solid Xe will be useful as a very sensitive $\mathrm{x}$-ray photocathode. The secondary electron emission yield $\delta$ was measured for condense layers of $\mathrm{Ne}, \mathrm{Ar}, \mathrm{Kr}$, and Xe excited by primary electrons of energy ranging from 0.05 up to $3 \mathrm{keV}$ [42]. The deposited thickness ranged from 0 up to a few thousands monolayers. The authors observed high yields of the secondary electrons. At $3 \mathrm{keV}$, the maximum yield was not attained for the thickest layers despite the fact that the measured yields exceed several tens (about 75, for solid Ar). Thus the rare gas solids are proven electron emitters and may be considered as competing with hydrogen-terminated diamond which is under extensive study both theoretically and experimentally. Disadvantage of the rare gas solids compared to the diamond is that the RGS need low temperature and vacuum conditions for preparation and operation of a cathode. On the other hand, the obvious advantage of using RGS is possibility of manufacturing cathodes of the orders of magnitude larger area compared to those fabricated using the diamond single crystal [43]. Hereupon, the RGS cathodes would be, undoubtedly, the most sensitive units. By way of example, one could think about a satellite orbiting the Earth and carrying solar-blind detectors designed with the use of the large area UV sensitive surfaces covered with a rare gas solid.

The present study proves that the electron emission from the RGS may be further significantly increased with an appropriate surface dopant.

1. Yu.A. Dmitriev, J. Low Temp. Phys. 150, 544 (2008).

2. Yu.A. Dmitriev, J. Low Temp. Phys. 158, 502 (2010).

3. E.V. Savchenko and Yu.A. Dmitriev, New Aspects of Relaxation Processes in Cryogenic Solids. In Applied Physics in the 21st Century, R.P. Valencia (ed.) Nova Science Publishers, New York (2010), Vol. 269.

4. Yu.A. Dmitriev, Phys. Cond. Matter B392, 58 (2007). 
5. Yu.A. Dmitriev, Fiz. Nizk. Temp. 35, 350 (2009) [Low Temp. Phys. 35, 265 (2009)].

6. G.B. Gumenchuk, A.N. Ponomaryov, A.G. Belov, E.V. Savchenko, and V.E. Bondybey, Fiz. Nizk. Temp. 33, 694 (2007) [Low Temp. Phys. 33, 523 (2007)].

7. Yufei Guo, M.C. Wrinn, and M.A. Whitehead, Phys. Rev. A40, 6685 (1989).

8. K. Rohr, J. Phys. B13, 4897 (1980).

9. M. Meunier and N. Quirke, J. Chem. Phys. 113, 369 (2000).

10. M. Meunier, N. Quirke, and A. Aslanides, J. Chem. Phys. 115, 2876 (2001).

11. A. Dalgarno and M.R.C. McDowell, Proc. Phys. Soc. London A69, 615 (1956).

12. I. Fisher-Hjalmars, J. Chem. Phys. 30, 1099 (1959).

13. H. Stewart and W. Massey, Negative Ions, Cambridge Monographs on Physics, Cambridge Univ. Pr. (1976).

14. J.K. Park, Internet Electron. J. Mol. Des. 4, 279 (2005), http://www.biochempress.com.

15. J.B. Cui, J. Ristein, and L. Ley, Phys. Rev. Lett. 81, 429 (1998).

16. R.A. Zhitnikov and Yu.A. Dmitriev, Fiz. Nizk. Temp. 24, 923 (1998) [Low Temp. Phys. 24, 693 (1998)].

17. T.V. Vorburger, B.J. Waclawski, and D.R. Sandstrom, Rev. Sci. Instrum. 47, 501 (1976).

18. V. Saile and E.E. Koch, Phys. Rev. B20, 784 (1979).

19. E.B. Saloman and C.J. Sansonetti, J. Phys. Chem. Ref. Data 33, 1113 (2005).

20. K.D. Bayes, D. Kivelson, and S.C. Wong, J. Chem. Phys. 37, 1217 (1962).

21. V.G. Storchak, D.G. Eshchenko, J.H. Brewer, S.P. Cottrell, S.F.G. Cox, E. Karlsson, and R.W. Wappling, J. Low Temp. Phys. 122, 527 (2001).

22. K.P. McKenna and A.L. Shluger, Nature Mater. 7, 859 (2008).

23. K.P. McKenna, P.V. Sushko, and A.L. Shluger, J. Am. Chem. Soc. 129, 8600 (2007).

24. K.M. Beck, M. Henyk, C. Wang, P.E. Trevisanutto, P.V. Sushko, W.P. Hess, and A.L. Shluger, Phys. Rev. B74, 045404 (2006).

25. K.M. Beck, A.G. Joly, O. Diwald, S. Stankic, P.E. Trevisanutto, P.V. Sushko, A.L. Shluger, and W.P. Hess, Surf. Science. 602, 1968 (2008).

26. E. Cheng, G. Ihm, and M.W. Cole, J. Low Temp. Phys. 74, 519 (1989).
27. G. Vidali, M.W. Cole, and J.R. Klein, Phys. Rev. B28, 3064 (1983).

28. G.A. Csáthy, J.D. Reppy, and M.H.W. Chan, Phys. Rev. Lett. 91, 235301 (2003).

29. R.A. Haefer, Kryo-Vakuumtechnik, Grundlagen und Anwendungen, Springer-Verlag, Berlin (1981).

30. V.B. Yuferov, Fiz. Nizk. Temp. 19, 579 (1993) [Low Temp. Phys. 19, 413 (1993)].

31. M. Frankowski, E.V. Savchenko, A.M. Smith-Gicklhorn, O. N. Grigorashchenko, G.B. Gumenchuk, V.E. Bondybey, J. Chem. Phys. 121, 1474 (2004).

32. E.V. Savchenko, I.V. Khyzhniy, S.A. Uyutnov, G.B. Gumenchuk, A.N. Ponomaryov, and V.E. Bondybey, Fiz. Nizk. Temp. 35, 520 (2009) [Low Temp. Phys. 35, 409 (2009)].

33. E.V. Savchenko, O.N. Grigorashchenko, A.N. Ogurtsov, V.V. Rudenkov, G.B. Gumenchuk, M. Lorenz, A. Lammers, and V.E. Bondybey, J. Low Temp. Phys. 122, 379 (2001).

34. E.V. Savchenko, I.V. Khyzhniy, S.A. Uyutnov, G.B. Gumenchuk, A.N. Ponomaryov, M.K. Beyer, and V.E. Bondybey, J. Phys. Chem. A115, 7258 (2011).

35. A.G. Belov, Fiz. Nizk. Temp. 25, 53 (1999) [Low Temp. Phys. 25, 40 (1999)].

36. A.S. Baryl'nik, A.I. Prokhvatilov, M.A. Strzhemechny, and G.N. Shcherbakov, Fiz. Nizk. Temp. 19, 625 (1993) [Low Temp. Phys. 19, 447 (1993)].

37. A.S. Baryl'nik, A.I. Prokhvatilov, and G.N. Shcherbakov, Fiz. Nizk. Temp. 21, 787 (1995) [Low Temp. Phys. 19, 607 (1995)].

38. V.G. Belan, N.N. Gal'tsov, A.I. Prokhvatilov, and M.A. Strzhemechnyı̆, Fiz. Nizk. Temp. 31, 1245 (2005) [Low Temp. Phys. 31, 947 (2005)].

39. Physics of Cryocrystals V.G. Manzhelii and Y.A. Freiman (eds.), AIP, Woodbury (1997).

40. R.A. Baragiola, M. Shi, R.A. Vidal, and C.A. Dukes, Phys. Rev. B58, 13212 (1998).

41. E.M. Gullikson, and B.L. Henke, Phys. Rev. B39, 1 (1989).

42. J. Cazaux, Y. Bozhko, and N. Hilleret, Phys. Rev. B71, 035419 (2005).

43. E. Wang, I. Ben-Zvi, X. Chang, Q. Wu, T. Rao, J. Smedley, J. Kewisch, and T. Xin, Phys. Rev. ST Accel. Beams 14, 061302 (2011). 